A.M. kpl.T.16, R/98g

$429176 \pi$

AUIGANAWERSTET IM. ADAMA MICKIEWICZA W POZNANIU

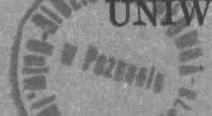
Lentive

\title{
STUDIA
}

\section{GERMANICA POSNANIENSIA}

\section{XVI}




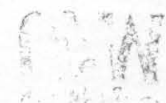

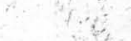

$$
\begin{aligned}
& \therefore \text { ? }
\end{aligned}
$$

$2 \quad 3$ ? $\because p$ 
- uNIWERSYTET IM. ADAMA MTCKIEWICZA W POZNANIU

\section{STUDIA \\ GERMANICA POSNANIENSIA}

\section{XVI}

Sprachwissenschaft

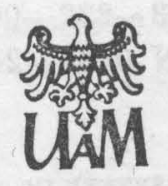

POZNAŃ 1989 


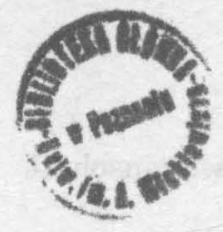

Redaktor: Anna Gierlińska

Redaktor techniczny: Michał Łyssowski

$$
\begin{gathered}
\text { ISBN } 83-232-0087-4 \\
\text { ISSN } 0137-2467
\end{gathered}
$$

WYDAWNICTWO NAUKOWE UNIWERSYTETU IM. ADAMA MICKIEWICZA W POZNANIU

Nakład 520+80 egz. Ark. wyd. 21,25. Ark. druk. 17,00+1 wkl. Papier druk, sat. kl. III. 80 g. $70 \times 100$. Oddano do składania 13 VIII 1987 r. Podpisano do druku w maju 1988 r. Druk ukończono w ezerweu 1989 r. Zam. nr $317 / 36$. Cena zl $660,-$

DRUKARNIA UNIWERSYTETU IM. ADAMA MICKIEWICZA W POZNANIU, UI. FREDRY 10.

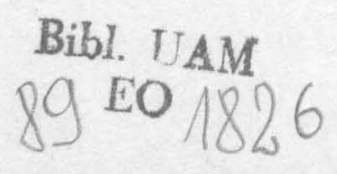




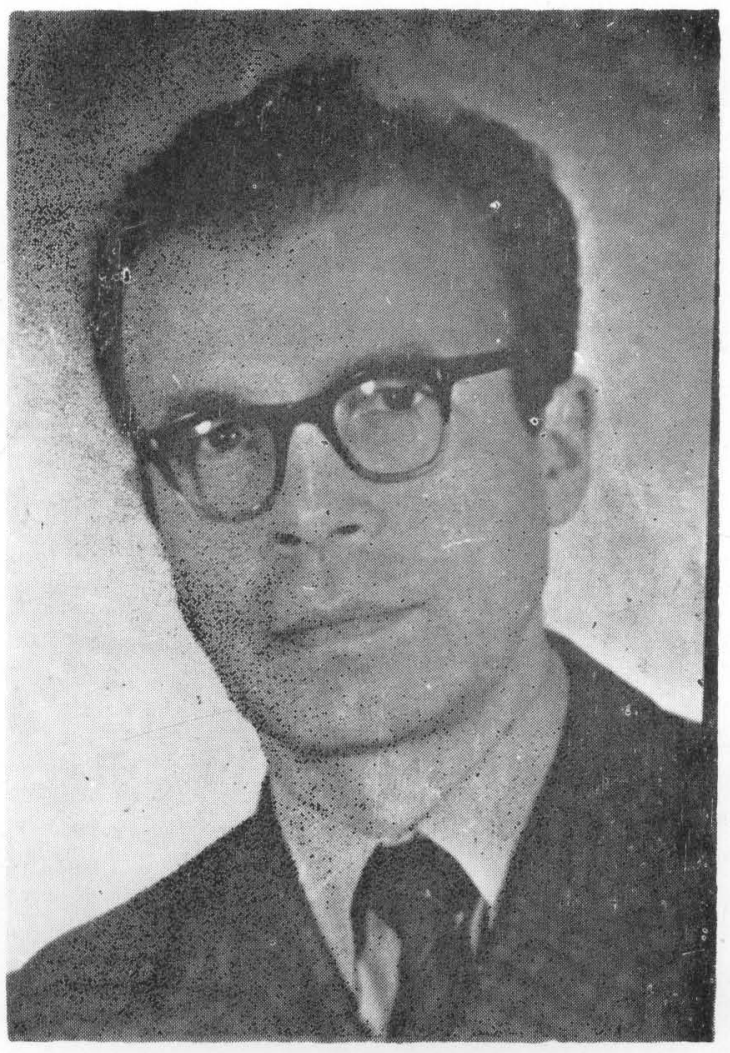

Freunde, Kollegen und Schüler widmen diesen Band

AndrzeJ Zdzisfaw Bzdęga

zu seinem 60. Geburtstag

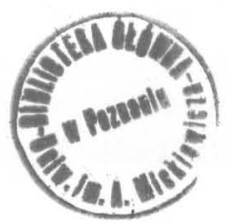





\section{INHALT}

ABHANDLUNGEN, AUFSÄTZE

Bolesław Andrzejewski (Poznań): Die Auswirkungen der Kritik der reinen Vernunft von I. Kant auf die Sprachphilosophie . . . . . . . . . . . . . . . . . . .

Sława Awedykowa (Poznań): Zum Bestand und Struktur der exozentrischen Konstruktionen im Norwegischen (bokmål) . . . . . . . . . . . . . . .

Jerzy Bańczerowski (Poznań): Aus philosophischen Problemen der Phonologie Józef Darski (Poznań): Die präskriptive Norm und die Entwicklungstendenzen in der Deklination des Substantivs im gegenwärtigen Deutsch . . . . . . .

Ulrich Engel (Mannheim): Wortklassen . . . . . . . . . . . . . . . . . . Janusz Figas (Poznań): Isomorphie bzw. Nichtisomorphie in der rezeptiven Verarbeitung zweitsprachlicher Äußerungen . . . . . . . . . . . . . . Alicja Gaca (Poznań): Artikelopposition im Deutschen und Artikellosigkeit im Polnischen aus der Sicht der Textstruktur . . . . . . . . . . . . . . Gerhard Helbig (Leipzig): Tendenzen und Probleme der neueren Partikel-Forschung . . . . . . . . . . . . . . . . . . . . . .

Gabriela Koniuszaniec (Poznań): Beobachtungen zum Kompositionstyp Substantiv + freundlich im Vergleich mit dem Polnischen . . . . . . . . . .

Dennis R. Preston (Ann Arbor, Michigan): The nicest English is in Indiana . . . Izabela Prokop (Poznań): Sprachhandlung Einladung/Vorschlag/Verabredung im Deutschen und Polnischen . . . . . . . . . . . . . . .

Czesława Schatte (Katowice): Phraseologisierte Partizipialkonstruktionen im Deutschen und Polnischen . . . . . . . . . . . . . . . . .

Jochen Schröder (Leipzig): Präfixverben in einer mehrstufigen Analyse - Versuch einer Modellierung unter konfrontativem Blick . . . . . . . . . . . . .

Wojciech Zdrojewski (Poznań): Wortbildung der deutschen Nomina instrumenti und ihre Äquivalente im Polnischen . . . . . . . . . . . . . . . . . 237

REZENSIONEN

Grammatische Studien - Beiträge zur germanistischen Linguistik in Polen. Göppingen 1985 (Izabela Prokop) . . . . . . . . . . . . . . . . Erwin Koller, Hans Moser (Hrsg.), Studien zur deutschen Grammatik. Johannes Erben zum 60. Geburtstag, Innsbruck 1985 (Andrzej Z. Bzdęga) . . . . . . Jahrbuch Deutsch als Fremdsprache, Band 9, München 1983 (Gabriela Koniuszaniec) . . . . . . . . . . . . . . . . . 
Wolfgang Fleischer (Hrsg.), Textbezogene Nominationsforschung. Studien zur deutschen Gegenwartssprache, Berlin 1985 (Hanna Jefremienko) . . . .

Czesława Schatte, Partizipialkonstruktionen im Deutschen und Polnischen, Katowice 1986 (Andrzej Z. Bzdęga) . . . . . . . . . . . . . . . . 262

Cathrine Fabricius-Hansen, Tempus fugit. Über die Interpretation temporaler Strukturen im Deutschen, Düsseldorf 1986 (Izabela Prokop) . . . . . . . .

Jahrbuch Deutsch als Fremdsprache, Band 10, München 1984 (Gabriela Koniuszaniec). . . . . . . . . . . . . . . .

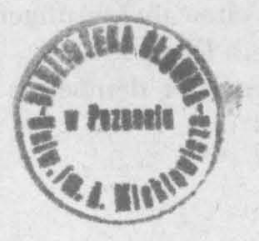




\title{
PRÄFIXVERBEN IN EINER MEHRSTUFIGEN ANALYSE - VERSUCH EINER MODELLIERUNG UNTER KONFRONTATIVEM BLICK
}

\begin{abstract}
Schröder Jochen, Präfixverben in einer mehrstufigen Analyse - Versuch einer Modellierung unter konfrontativem Blick [Multistage analysis of prefixal verbs an attempt at a model of description in confrontative aspect], Studia Germanica Posnaniensia, Adam Mickiewicz University Press, Poznań, vol. XVI: 1989, pp. 223-235, ISBN $83-232-0087-4$, ISSN $0137-2467$.
\end{abstract}

In reference to requirements and needs of foreign language teaching the author, motivated by the requirements and needs of methodology of foreign language teaching, performs a confrontative analysis of prefixal verbs of the German language and their Polish equivalents and proves that word-formation studies must be extended with a syntactic aspect since inter-language differences are manifested in an actual usage (on the surface structure).

Jochen Schröder, Herder Institute, Karl Marx University, Leipzig, German Democratic Republic.

1. Dem Verb als satzbildendem Zentrum (vom Standpunkt der Valenztheorie her) und/oder aber als wesentliche Konstituente des Satzes (traditionelle Grammatik wie auch Konstituentenstrukturanalyse) ist eine besondere Aufmerksamkeit in linguistischen Untersuchungen zur Fundierung des Fremdsprachenunterrichts zu schenken. Nun ist es aber wohl so, daß lexikalische Einheiten dem eine Sprache Erwerbenden in der Regel eingebunden in die syntaktische Oberfläche begegnen. Dies gilt auch für solche Einheiten, denen wir uns hier zuwenden wollen, den präfigierten Verben. Sie sind aufgrund ihrer nur ihnen eigenen Strukturiertheit wie auch wegen ihrer relativ 
großen Häufigkeit im Deutschen in besonderem Maße bei der linguistischen Beschreibung zu berücksichtigen. Immerhin gibt G. MUNGAN an, daß die Zahl verbaler Simplizia nach E. MATER 3205 beträgt, von denen 1581 weder präfigiert noch zusammengesetzt werden. Von den restlichen 1624 Simplizia werden mit denen für das Deutsche typischen Mitteln der Zusammensetzung und Präfigierung 11356 neue Verben gebildet (MUNGAN 1986: 24). Nun eröffnen Zusammensetzungen, ohne zu verkennen, daß es hier auch Ausnahmen gibt, aufgrund ihrer größeren Bedeutungshaltigkeit beider Komponenten leichter Möglichkeiten für die semantische Erschließung als Präfigierungen, in denen das unselbständige Wortbildungsmorphem bedeutungsärmer oder aber, anders gesehen, von weiterer, unspezifizierterer Bedeutung (Polysemie) ist. (Vgl. FLEISCHER/STEPANOWA 1985: 68, 136 ff.) Für beide Verfasser sind die im Zentrum der Kategorie befindlichen Wortbildungsmorpheme gekennzeichnet durch:

1. Reihen-, Serienbildung nach einem semantisch bestimmten Modell,

2. Ausprägung einer Wortbildungsbedeutung,

3. Gebundenheit des Morphems (ebd. 68).

Bei den präfigierten Verben können wir mit M. D. STEPANOWA/W. FLEISCHER (ebd. 137 ff.) unterscheiden:

Gruppe III - Bildungen mit fehl-, los-, weg-..., die generell trennbar auftreten;

Gruppe II - Bildungen mit nachweisbarer Bezugnahme auf präpositionale/adverbiale Einheiten, die trennbar und seltener untrennbar auftreten, z. B. ab-, an-, auf-, aus... ;

Gruppe I - Generell untrennbare Wortbildungskonstruktionen mit den eigentlichen Präfixen ent-, er-, be-, ver-, zer-.

Die letztgenannte Gruppe, die wir im folgenden Präfixverben nennen wollen, scheint uns von besonderem Interesse, sind doch für sie o. g. Kriterien in Gänze zutreffend, und sind sie vom Standpunkt der Synchronie nicht mehr auf ein morphematisch ähnliches Bezugselement in der Gegenwartssprache zu beziehen, und sind sie, was für das Fremdsprachenerlernen noch schwerer wiegt, so beschaffen, daß sie über eine Reihe von Semen verfügen, von denen nur einige konstant sind und gleichzeitig realisiert werden, während andere potentiell (latent) sind, woraus sich ihre Polysemie ergibt (vgl. ebd. 100).

Gerade diese Polysemie aber ist es, die den ausländischen Lernenden im allgemeinen und je nach muttersprachlicher Bezugnahme in unterschiedlicher Weise Schwierigkeiten bereitet. Und dennoch kann man wohl nicht annehmen, daß diese Präfixverben so wie die Simplizia gespeichert werden, eher, daß der normale Sprachteilnehmer, also auch der die deutsche Sprache in angemessener Weise benutzende Nichtmuttersprachler ,die tausenden vorkom- 
menden oder möglichen Zusammensetzungen und Ableitungen nicht beherrschen (könnte), wenn er sie alle als fertige, unteilbare Lexeme im Gedächtnis behalten müßte, um sie bei Bedarf nur anzuwenden." (POLENZ 1968: 13) Anders ausgedrückt: „,Der Sprecher verfügt in seinem Sprachschatz über ein Arsenal von angelernten generativen Typen, wodurch sich der größte Teil des Systems erschließt und mit dessen Hilfe er selbst Prägungen vornehmen kann." (HUNDSNURSCHER 1968: 210) Das gilt um so mehr dann, wenn solche Präfixe wie in Gruppe I noch produktiv sind. Andererseits ist festzustellen, daß mit Ausnahme der Arbeiten von G. ZIFONUN 1973; H. GÜNTHER 1974 und G. MUNGAN 1986 wenig konsistente Analysen speziell zu den Präfixverben zur Verfügung stehen, die den Anforderungen der modernen Sprachwissenschaft genügen könnten. Und selbst diese Arbeiten zielen in ihrer Art auf bestimmte Erscheinungen dieser Verbgruppe, die schon traditionell im Blickpunkt der Untersuchung standen. Sie sind meist der Wortbildungstheorie verhaftet, weniger der Grammatik, so daß dann der Blick auf die Zusammenhänge zwischen .den verschiedenen Präfigierungen gerichtet wird (ZIFONUN), auf die Ornativität (GÜNTHER) oder aber auf die semantische Interaktion ohne Berücksichtigung der syntagmatischen Beziehungen (MUNGAN). Es scheint uns erforderlich, gerade auch bei den Präfixverben mit zu berücksichtigen, was A. Z. BZDĘGA 1984: 3, so formuliert: „Die syntaktische Beschreibung und Interpretation von Verben schließt neben der kasusabhängigen Kookkurrenz deren semantische Restriktionen ein, die in der Syntax und Lexikologia mit verschiedenen Termini erfaßt werden: semantische Kongruenz (LEISI), lexikalische Solidarität (COSERIU), Kollokation (JOOS), syntaktisches Feld und wesenhafte Bedeutungsbeziehungen (PORZIG), semantisch-syntaktischer Hof (GREBE)." Einen Ansatz für eine Beschreibung von Verben (also auch für Präfixverben) sehen wir in G. HELBIGs 1983 unterbreitetem Vorschlag einer mehrstufigen Beschreibung. Wir wollen uns hier auf die Stufe der logischen Struktur, die Stufe der semantischen Kasus und die Stufe der syntaktischen Wertigkeit beschränken, wobei wir nicht verkennen, daß zu einer vollständigen Beschreibung auch die zwischengeschalteten Stufen mit den valenzrelevanten und valenzirrelevanten Merkmalen des Prädikats sowie die semantischen Merkmale der Substantive gehören, weil sonst bestimmte Restriktionen bzw. Differenzierungen nicht manifest gemacht werden können. Es kann nicht Anliegen dieser Studie sein, eine umfassende Analyse der Präfixverben zu geben, vielmehr soll an ausgewählten Gruppen gezeigt werden, wie entsprechende Untersuchungen aussehen könnten.

2. Aus der großen Zahl der be-Vérben greifen wir nur solche heraus, die nach erfolgter Präfigierung das Merkmal [loc motio] behalten. Scheint die logische Struktur noch relativ einheitlich wiedergegeben werden zu können 
als $R(a, b)$, so treten schon auf Stufe II (semantische Kasus) interpretative Schwierigkeiten auf. U. BRAUSSE 1980: 28 ff., ordnet den zweiwertigen Verben treten - betreten, steigen - besteigen, fahren - befahren... die Kasusrollen AGENS LOCATIV zu, während H. GÜNTHER 1974: 138, z. T. dieselben Verben mit der Kasusstruktur AGENS INSTRUMENT AFF zu einer geschlossenen Gruppe mit Subgruppen für befahren, befliegen, bereisen, besegeln... sowie für besteigen und betreten zusammenfaßt. Dazu stellt er fest: „Es handelt sich um echte Präfixbildungen (...) Sa ist in dieser Gruppe stark lokativ und bezeichnet stets ,Landschaft, Gegend, Strecke" . Dieser verbalen Einschätzung ist zuzustimmen. Sie deckt sich aber nicht mit den angegebenen Kasus, wird doch hier kein Objekt affiziert, sondern AGENS (oder aber ein als selbständig agierend aufgefaßtes INSTRUMENT, und nur dies wollen wir aus Gründen, auf die wir hier nicht näher eingehen können, berücksichtigen) erreicht mittels einer Fortbewegung etwas, was mit LOCUS (DIR) wiederzugeben wäre, oder aber bewegt sich auf einem solchen LOCUS (PATH) fort. Daraus ergeben sich zwei unterschiedliche Beschreibungsansätze für diese Verben:

Für

(1) Er befährt (mit seinem Wagen)/zum ersten Mal/ die neue Straße.

(2) Er bereist das Land.

(3) Die Fluggesellschaft befliegt die Linie nach W. gilt:

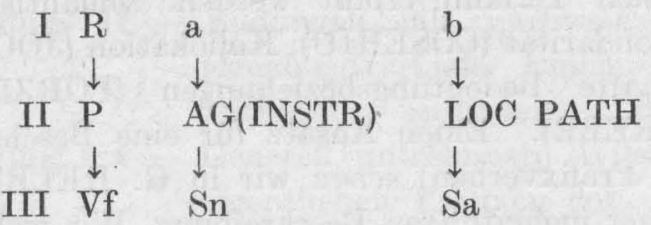

Für

(4) Er besteigt den Berg / das Pferd / den Thron / das Flugzeug.

(5) Er betritt das Zimmer.

(6) Er befährt ${ }_{2}$ den Schacht. (bergm.) gilt:

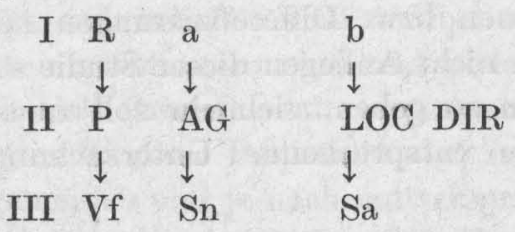

Die Wörterbuchangaben zu den polnischen Entsprechungen zeigen, daß nur die Annahme eines in sich differenzierten LOCATIVs der Überführung von der einen Argumentenstelle bis an die Oberfläche (deutsch Sa) gerecht 
werden kann; während im Polnischen mit verschiedenen Ausprägungen von $\mathrm{pS}$ zu rechnen ist, fehlt doch hier ein dem dt. be- vergleichbares Element, das Verben o. g. Art transitiviert. Das erklärt zugleich, daß für die polnischen Äquivalente zu $(1-3)$ als Verben im Normalfall Simplizia wie jeździé, żeglować, podróżować, lataé auftreten, die den zweiten Aktanten als pS realisieren, und zwar in der für LOC STAT typischen Form mit dem syntaktischen Kasus Lokativ, der aber hier als LOC PATH aufzufassen ist, was durch die Möglichkeit bzw. Obligatheit der Präpositionswahl po anstelle des na bei offensichtlicher Distribution deutlich wird. Dem für deutsch $(1-3)$ aufgestellten Modell steht so ein polnisches Modell gegenüber:
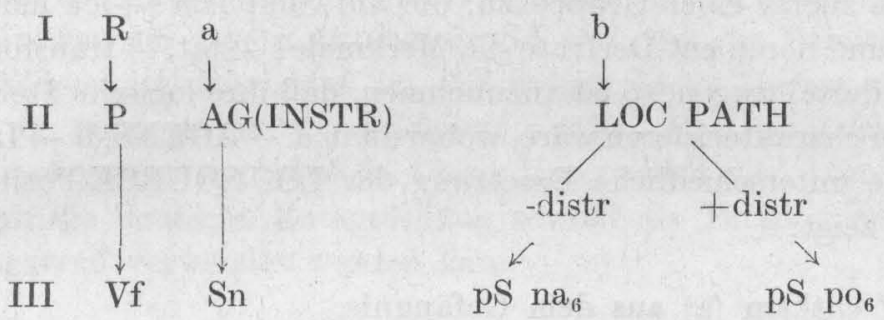

Für das für (4-6) entwickelte Modell finden wir mit wchodzić, wstępować, wspinać się, wsiadać mit entsprechenden Restriktionen für das zu wählende Substantiv und schließlich mit zjeżdżać do szybu für das bergmännische einen Schacht befahren Verbøn, die durch solche Vorsilben gekennzeichnet sind, die wir an anderer Stelle (vgl. SCHRÖDER 1983, 213 ff.) für das Deutsche Adverbialzusätze genannt haben, was auch für solche polnische Vorsilben mit direktem Bezug zu Präpositionen gelten könnte, ohne daß dabei verkannt wird, daß hier bestimmte Aspektregularitäten hinzukommen. Bezeichnend ist, daß zu solchen Adverbialzusatzverben der 2. Aktant ebenfalls als p̂S realisiert wird, aber eben mit Präpositionen wie do, das in lokaler Verwendung von sich aus auf eine Direktion zielt, oder aber $\mathbf{n a}_{4}, \mathbf{w}_{4}$, die ihre direktive Wirkung über die Kasusopposition Akkusativ: Lokativ erreichen, hier also nur Akkusativ erlauben. Daraus ergibt sich entsprechend dem Modell für die deutschen Sätze $(4-6)$ für das Polnische:

$\begin{array}{rlll}\text { I } & \mathrm{R} & \mathrm{a} & \mathrm{b} \\ & \downarrow & \downarrow & \downarrow \\ \text { II } & \mathrm{P} & \mathrm{AG} & \text { LOC DIR } \\ & \downarrow & \downarrow & \downarrow \\ \text { III } & \stackrel{\mathrm{V} f}{\mathrm{Sn}} & \mathrm{S} & \mathrm{pS} \mathrm{do} \mathrm{do}_{2}, \mathrm{na}_{4}, \mathrm{w}_{4}\end{array}$

Aus dem erstgenannten Modell (poln.) fällt heraus objeżdżać okolicę (Sn, Sa), aus dem zweiten dosiadać konia $(\mathrm{Sn}, \mathrm{Sa})$. Beiden Verben würden die entsprechenden deutschen Modelle genügen. 
2.1. Versuchen wir, das hier an einer relativ kleinen Gruppe von be-Verben entwickelte Verfahren auf seine Verwendbarkeit bei einer umfangreicheren, aber auch anscheinend semantisch deutlicher gekennzeichneteren Gruppe von ent-Verben zu überprüfen, so stoßen wir bei einem Blick in zweisprachige Wörterbücher sofort auf die Schwierigkeit, daß wir, ohne daß Vollständigkeit angestrebt werden könnte, deutschen ent-Verben ohne Berücksichtigung der polnischen Simplizia zumindest folgende polnische Präfix-/Adverbialzusatz-Verben zuordnen können: od-, roz-, u-, wy- und za-Verben. Kann dennoch auch bei einer solchen Vielfalt der von uns gewählte Ansatz (vgl. SCHRÖDER 1985) zu tragbaren Ergebnissen führen?

Wenden wir uns zuerst einer Gruppe zu, die auf Simplizia [+loc motio] zurückzuführen ist und deren ent-Derivate die Merkmale [- stat, +transform, +ingressiv, +mov deloc] tragen, so ist anzunehmen, daß ihre logische Stelligkeit durch $\mathrm{R}(\mathrm{a}, \mathrm{b})$ zu charakterisieren wäre, wobei dann $a \rightarrow$ AGENS, $b \rightarrow$ LOC SOURCE wäre. Die unterschiedliche Besetzung der LOC-SOURCE-Position an der Oberfläche zeigt:

(7) Er entfloh / entkam (a) aus dem Gəfängnis

(b) seinen Verfolgern

(c) der Gefahr

Zumindest tendenziell läßt sich im Deutschen feststellen, daß pS nur dann erhalten bleibt, wenn der verlassene Bereich deutlich lokale Konturen zeigt und vor Beginn des im Satz wiedergegebenen Geschehens den Handelnden einschließt [+spatial claus]. Er erscheint als Sd, wenn eine Person [+hum] Zentrum der Lokalisation ist oder aber ein Abstraktum als Veranlassung für die Handlung des AGENS gesehen wird. Entsteigen ( $\mathrm{Sn}, \mathrm{Sd}$ generell) ausgenommen, das mit besteigen korrespondiert, gilt für die ganze Gruppe:

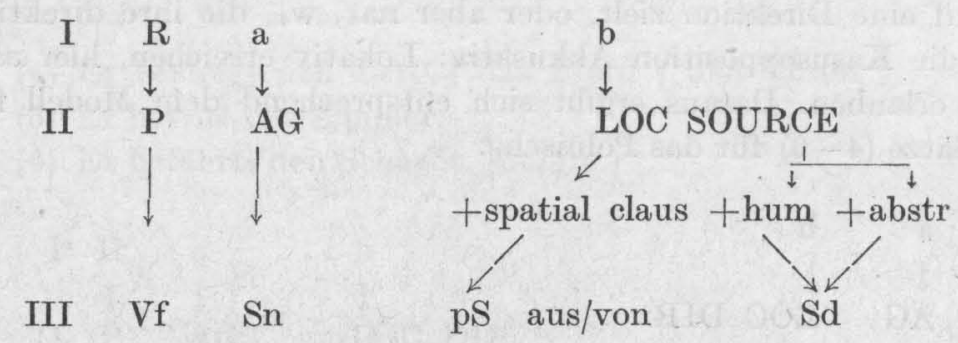

Dieser deutlich abzugrenzenden Gruppe entsprechen im Polnischen zumeist $\mathbf{u}$-Verben, deren Kern uciekać und uchodzić darstellen dürften. Die Besetzung der b-Stelle an der Oberfläche zeigt Unterschiede zum Deutschen. Diese Unterschiede sind nach Wörterbucheintragungen anzusetzen als: 


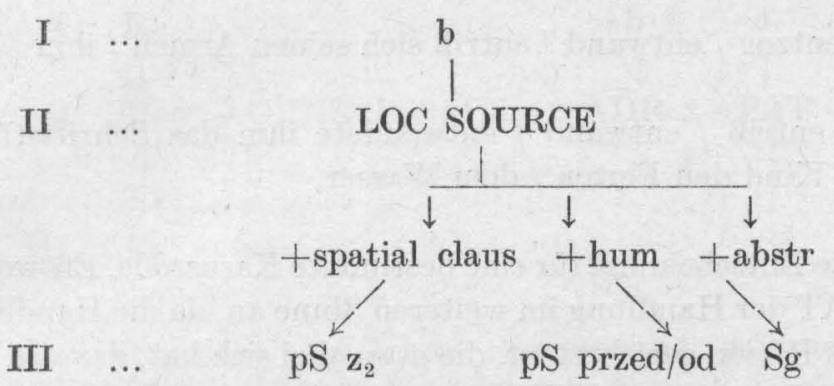

Ist der Aktant Sn auf einen Vorgangsträger (VT) zurückzuführen, wird im Deutschen der zweite Aktant als Sd realisiert, im Polnischen als pS mit $\mathbf{z}_{2}$. Auffallend ist dabei, daß im Polnischen solche Verben zumeist nicht mehr mit u-, sondern mit wy- präfigiert werden. Man vergleiche entfallen/entgleiten - wypadać/wymykać się. Dieser Unterschied wird dann besonders deutlich, wenn die deutsche Entsprechung sowohl als Tätigkeits- wie auch als Vorgangsverb verwendet werden kann:

(8) Er entwich/entsprang aus dem Gefängnis.

Uciekal z więzienia.

(9) Gas entwich (dem Rohr).

Gaz wydobywał się (z rury).

(10) ?Wassser entwich / entströmte (dem Rohr).

Woda wyciekała (z rury).

(11) Der Fluß entspringt in den Bergen (LOC STAT).

Rzeka wypływała z gór (LOC SOURCE).

(12) Dieser Plan entsprang dem Bedürfnis nach Frieden.

Plan ten wyplywal z potrzeby pokoju.

Die Ursache dafür, daß hier wy- auftritt, liegt aber weniger in der Differenzierung Tätigkeit - Vorgang, sondern in der deutlicheren LOC-SOURCE-Beziehung (heraus) des Adverbialzusatzes wy-, denn auch uchodzić/uciekać lassen sich in Sätzen wie Czas uchodzi/ucieka - Die Zeit entflieht finden. Das Grundmodell dieser Verben ist, abgesehen von der Unterscheidung in VT und $\mathrm{AG}$ und deren Konsequenzen und bei ersterem möglicher Fakultativität des LOC SOURCE (vgl. $(9,10)$ ) sowie der spezifischen Besetzung im Deutschen in (11) o. g. Modell unter Berücksichtigung der Modifikationen und Restriktionen gleich.

Auf logischer Dreistelligkeit beruht die syntaktische Dreiwertigkeit bei entziehen, entreißen, entringen, entwinden, wobei die neu hinzukommende dritte Stelle bei Eigenbezug mit sich $_{4}$ wiedergegeben wird: 
(13) Sie entrang / entzog / entwand / entriß sich seinen Armen / ihm / seinem Einfluß.

(14) Sie entrang / entriß / entwand / entwendete ihm das Schriftstück.

(15) Sie entriß das Kind den Fluten / dem Wasser.

Schwierig ist hier die Entscheidung für eine bestimmte Kasusrolle. Entweder man nimmt ADRESSAT der Handlung im weiteren Sinne an, da die Handlung des AGENS ja auf eine Person gerichtet ist, die etwas bei sich hat, das von der Handlung direkt betroffen wird (PATIENS), oder aber (und dafür spricht die syntaktische Umgebung der zum Vergleich gewählten polnischen wy-Verben mit pS) LOC SOURCE. Dafür spricht, daß es möglich ist, die Person ebenso im Zentrum der Lokalisation dessen zu sehen wie das PATIENS. Daraus ergäbe sich folgendes Modell:

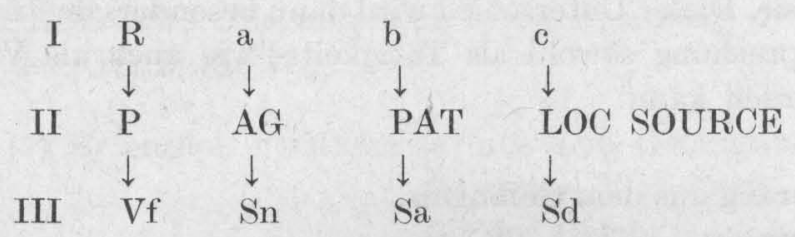

Zu diesem Typ würden auch gehören: entnehmen, entleihen, entlocken. Auch hier zeigen die polnischen Entsprechungen wyjmować, wypożyczać, wydobywać, wyludzać, wyciskać mit ihren unterschiedlichen Restriktionen bei der Besetzung der LOC-SOURCE-Position die pS, nicht Sd.

Für das Deutsche könnte hier entziehen einbezogen werden. Andererseits bildet es quasi ein Orientierungsverb für eine ganze Gruppe, die keine lokale Komponente mehr enthält und wo die valenzrelevanten Merkmale anzusetzen sind als [-stat, +transform, +aktional], [+transform] wäre zusätzlich zu spezifizieren als [+egressiv]. Muß bei entziehen noch genannt werden, was entzogen wird, woraus sich logische Dreistelligkeit ergibt, so wird bei den anderen Verben das zu Entziehende in das Verb inkorporiert (logische Dreistelligkeit, aber syntaktische Zweiwertigkeit). Es besteht Modellgleichheit mit letztgenanntem Modell, wenn LOC SOURCE durch ADRESSAT 2 ersetzt wird. Bei Inkorporierung des PATIENS in das Verb könnte der bisherige ADRESSAT 2 als PATIENS angesehen werden. Man vergleiche:

(16a) Das Mittel (INSTR) entzog dem Stoff (ADR 2) die Farbe (PAT). (16b) Das Mittel entfärbte den Stoff. Srodek odbarwiał tkaninę.

Das Modell für diese deutschen ent- und polnischen od-Verben ist, abgesehen von Lücken bzw. Réestriktionen, gleich: 


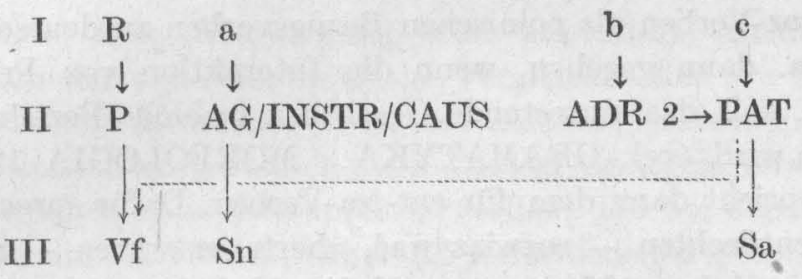

Man vergleiche: entfärben - odbarwiać, entfetten - odtluszczać, entkalken - odwapniać... Dabei darf nicht übersehen werden, daß z. B. entfärben bei [+hum] als Vorgangsverb zblednąć - erblassen, bei Blättern zżółknąć gelb werden verlangt und beispielsweise entmündigen wiederzugeben ist als oddawać pod kuratelę oder ubezwłasnowolnić, Unterschiede, die ebenso wie bei den anderen Gruppen noch an einer Reihe anderer Fälle aufzuzeigen wären. Dies spricht jedoch nicht gegen die Annahme einer Kerngruppe von entziehen-Verben mit Inkorporation des PATIENS mit Entsprechung über drei Stufen für ent- und od-Verben.

Eine relativ kleine Gruppe von ent-Verben findet ihre Entsprechung in za-Verben. Sie sind zu charakterisieren als [-stat, +transform, +aktional], wobei [+transform] durch [+inchoativ] zu spezifizieren wäre. Im Prozeß des Geschehens entsteht etwas, was vorher in der Art nicht vorhanden war (RESULTAT). Wenn wir von Vorgangsverben absehen, läßt sich für Sätze wie

(17) Der Heizer / Der Wind entfachte / entzündete das Feuer.

annehmen:

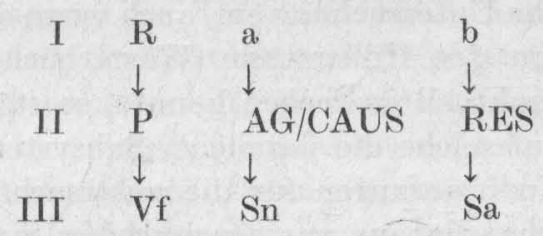

Die Vorgangsverben der gleichen Gruppe sind in der Regel einwertig:

(18) Er entschlief.

(19) Das Feuer entflammte.

Die polnischen Verben dieser Gruppe genügen den gleichen Bedingungen, wobei allerdings $\mathrm{zu}$ berücksichtigen ist, daß bei übertragenem Gebrauch eine Auffüllung sowohl innerhalb des Modells als auch wegen anderer lexikalischer Beziehungen über das Modell hinaus zu berücksichtigen ist. Das gilt schon für die Entsprechung zu entfachen - rozniecać. 
Das Eintreten von roz-Verben als polnischen Bezugsverben zu deutschen ent-Verben scheint v. a. dann gegeben, wenn die Interaktion von Präfix und Stamm dazu führt, daß das eintretende Geschehen in einer Beziehung zu, auseinander' gesehen wird (vgl. GRAMATYKA... MORFOLOGIA 1984: $504 \mathrm{f}$.). Das Modell entspricht dann dem für ent-/za-Verben. Dafür sprechen solche Verben wie ök. entflechten - rozwiązywać, übertr. entwirren - rozplątywaé, entrollen - rozwijaé, entfalten - rozwijać/rozkladać, mar. rozpinać u. ä., was auch die Beziehung entfachen - rozniecać erklären könnte. Problematischer wird das schon bei enthärten - rozhartować oder entminen - rozminować, wo man eigentlich nach $\mathrm{o}$. g. Modell od- erwarten müßte.

Solche Überlappungen in Grenzbereichen von Untergruppen zeigen zugleich, daß Präfixe und, wenn auch in geringerem Maße, Adverbialzusätze schon in einer Sprache nur mehr oder weniger geschlossene Systeme von Ableitungen erstellen können. Im Rahmen des jeweiligen sprachlichen Systems werden über den Sprachgebrauch und damit über richtige, bedingt richtige und sogar falsche Analogiebildungen, die über Usus dann im Laufe der Zeit zur Norm werden können, neue lexikalische Einheiten geschaffen. Sie sind dann (auf unseren hier dargestellten Gegenstandsbereich bezogen) nur mehr formal als Präfixverb anzusetzen, aber von der Synchronie her nicht mehr in ihre semantischen Bestandteile nach dem Muster Präfix - Stamm zu zerlegen. Oft hat Bedeutungsübertragung daran ihren ganz spezifischen Anteil. So ist beispielsweise das in verschiedenen Bedeutungen existente sehr frequente Verb entwickeln nicht mehr in seiner konkreten Bedeutung im Gebrauch:

(20) *Er entwickelte die Binde, die sie um das Handgelenk hatte.

Bezieht man nun eine zweite Sprache in die Untersuchung ein, auch wenn das hier nur schon vom Korpus und den benutzten Hilfsmitteln (Wörterbücher) her marginal und mehr oder weniger punktuell geschehen konnte, so stößt man in der zweiten Sprache ebenfalls auf solche die jeweiligen Subsysteme sprengenden Grenzen und kommt zu Modifizierungen für die untersuchten Gegenstandsbereiche in der ersten Sprache, die aus nur einsprachiger Sicht dem Blick verschlossen bleiben mußten (vgl. dazu die vorgelegte Beschreibung der ent-Verben bei SCHRÖDER 1985). Dennoch sind auch solche einsprachigen Analysen für die Stoffaufbereitung dieser Sprache als Fremdsprache von Wert, wenn sie zumindest einen Versuch darstellen, konfrontationsfreundlich angelegt zu sein. Denn: „Eine Schwierigkeit auf dem Wege zur kontrastiven Regelbildung stellt das Wissen von den Regeln der zu vergleichenden Sprachen dar. Im Idealfall entfällt die Phase der einzelsprachigen Regelbildung. Meistens liegen aber keine ausgewogenen, mit dem Tertium comparationis konformen Beschreibungen, von $\mathrm{L}_{1}$ und $\mathrm{L}_{2}$ vor, sie müssen erst nach (...) methodischen Grundsätzen miteinander abgestimmt werden" (BZDĘGA 1985: 7). 
Gerade diese Abstimmung wird erleichtert, wenn schon einzelsprachige Untersuchungen eine mögliche Vergleichbarkeit (in Sprachtheorie, Methodologie und Terminologie) zu berücksichtigen suchen, also in diesem Sinne konfrontationsfreundlich sind. Diesem Anliegen versuchten wir hier gerecht zu werden, ohne behaupten zu wollen, daß bei der Polysemie des ent-Präfixes, die nicht zuletzt über die verschiedenen polnischen Möglichkeiten der Entsprechung nachgewiesen werden konnte, nicht eine gründlichere Untersuchung zu noch differenzierteren Ergebnissen führen würde und an bestimmten Stellen auch zur Falsifizierung mehr oder weniger hypothetischer Ansätze.

2.2. Konfrontative Ansätze lassen sich dann leichter gewinnen, wenn der Systemausschnitt, der konfrontativ betrachtet wird, sowohl syntaktisch als auch semantisch eng begrenzt ist. Diesen Fall haben wir in einer letzten Gruppe vor uns, den zer-Verben der Tätigkeit. Nach G. MUNGAN 1986: 193 f., herrscht bei den zer-Verben die Bedeutung des Teilens, Auseinanderlegens und Zerkleinerns sowie des Beschädigens und Unansehnlichmachens vor. SCHATTE 1985: 40, bemerkt zu dem Satz

(21) Sie zerquetscht die Kartoffeln zu Brei.

Ona rozgniata ziemniaki na miazge.

daß er „eine Annihilation von (zählbaren) Kartoffeln und eine Kreation von (unzählbarem) Brei darstellt", nach unserer Definition wäre die Kreation ein RESULTAT. Typisch erscheint die Wiedergabe des zer-Verbs mit einem roz-Verb. Nach den Feststellungen zu den ent-/roz-Verben (vgl. 2.1.) können wir davon ausgehen, daß sich die zer-Verben zu den roz-Verben wie eine Teilmenge zu einer Gesamtmenge verhalten. Wir können unter Vernachlässigung des INSTRUMENTs annehmen, daß die deutschen zer-Verben und die entsprechende Teilmenge der polnischen roz-Verben charakterisiert sind durch [-stat, +transform, +aktional], [transform] wäre durch [+resultativ] zu spezifizieren, sie sind auf die logische Stelligkeit von $R(a, b, c)$ zurückzuführen. Dabei gilt, daß das RESULTAT an der Oberfläche nur dann als fakultativer Aktant erscheinen kann, wenn es als zählbare Menge von Einzelstücken [+division] oder aber als eine neu erstandene, aber nicht zählbare Menge [+dekomp] auftritt, nicht aber, wenn es durch die Tätigkeit zu einer nicht mehr erfaßbaren Menge von Einzelstücken wird [+deform]. Beispiele für die drei unterschiedlichen Gruppen wären: zerschneiden (in Stücke), zermahlen (zu Staub), zerstören / zertrümmern (*in/zu ...). Lediglich an der Oberfläche differieren die entsprechenden polnischen Verben in ihrer Umgebung von den deutschen, scheint doch hier in der Regeln der Unterschied in der Besetzung der Präposition in der fakultativen pS-Stelle nicht gemacht zu werden. So ergibt sich für diese Gruppe der zer-/roz-Verben folgendes Modell: 


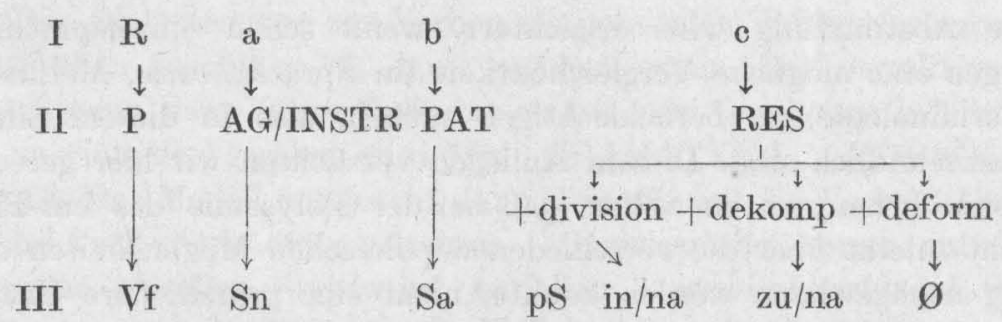

3. In vorgelegter Studie wurde versucht, den Nachweis zu erbringen, daß die Untersuchung der Präfixverben nicht nur Sache der Wortbildung sein darf, sondern gerade für die Anforderungen des Fremdsprachenunterrichts der syntaktischen Umgebung dieser Verben die gebührende Aufmerksamkeit zu schenken ist, wenn die Linguistik entsprechende Vorleistungen für die Verbesserung dieser Unterrichtsdisziplin erbringen will, indem sie diesen Regularitäten nachgeht. Wir nehmen an, daß man mit einem mehrstufigen Ansatz der Untersuchung von Präfixverben sowohl in einzelsprachigen als auch in (in Ansätzen versuchten) konfrontativen Untersuchungen zu Ergebnissen kommt, die sowohl Gemeinsamkeiten auf tieferliegenden Ebenen als auch untersehiedlichen Ausprägungen auf der Oberfläche besser gerecht werden kann als das bisher bei nur auf die Wortbildung orientierten Untersuchungen der Fall sein konnte. Das ist schon von Bedeutung, denn: „Fast alle zwischensprachlichen Kontraste treten in den Oberflächenstrukturen auf. Eine Ausnahme bilden nur Kontraste im .Bereich solcher kategorialer Inhaltsmomente, die nur in der einen Sprache formalisiert vorkommen, in der anderen also, mögen sie auch im Satzzusammenhang irrelevant erscheinen, einen Informationsverlust bedeuten..." (BZDEGGA 1985: 7). Wenn auch dieser Frage nicht im Detail nachgegangen werden sollte, so ist doch anzunehmen, daß die systemhaft angelegten Präfigierungen im Deutschen mit ihren spezifischen syntaktischen Reflexen eine besondere Form der Formalisierung darstellen kann, der im Polnischen andersartige Bildungen (z. B. wy-) ohne vergleichbare syntaktische Reflexe wie auch gleichartige Bildungen (z. B. roz-) gegenüberstehen.

\section{LITERATUR}

Brauße, U., Semantische Probleme konfrontativer Valenzuntersuchungen (Deutsche Simplex- und be-Verben und ihre französischen Äquivalente), LS/ZISW A 69/I, Berlin 1980.

Bzdęga, A. Z., Sog. kommodische und inkommodische Syntagmen im Deutschen und Polnischen, SGP XIII, Poznań 1984.

Bzdęga, A. Z., Induktion und Deduktion in der Erstellung von kontrastiven Grammatiken, LAB 48, Léipzig 1985. 
Bzdęga, A. Z.; Chodera, J.; Kubica, S., Handwörterbuch Polnisch-Deutsch, Warszawa 1973.

Chodera, J.; Kubica, S., Handwörterbuch Deutsch-Polnisch, Warszawa 1966.

Gramatyka wspótczesnego języka polskiego. Morfologia, Warszawa 1984.

Günther, H., Das System der Verben mit be-in der deutschen Sprache der Gegenwart. Ein Beitrag zur Struktur des Lexikons der deutschen Grammatik, als: Linguistische Arbeiten 23, Tübingen 1974.

Helbig, G., Valenz und Lexikographie, DaF 3/1983.

Hundsnurscher, F., Das System der Partikelverben mit aus in der Gegenwartssprache, als: Göppinger Arbeiten zur Germanistik 2, Göppingen 1968.

Mungan, G., Die semantische Interaktion zwischen dem präfigierenden Verbzusatz und dem Simplex bei deutschen Partikel- und Präfixverben, Frankfurt a. M./Bern/New York 1986.

Polenz, P. v., Ableitungsstrukturen deutscher Verben, Zeitschrift für deutsche Sprache $1-2 / 1968$.

Schatte, Chr., Die Semantik kausativer Sätze dargestellt am Deutschen und Polnischen,. Katowice 1985 .

Schröder, J., Fortbewegungsverben als Beschreibungsproblem (Simplicia - Adverbial-. zusätze - be-Präfixe), DaF 4-5/1983.

Schröder, J., Ent-Verben in einer mehrstufigen Analyse, DaF 6/1985.

Stepanowa, M. D.; Fleischer, W., Grundzüge der deutschen Wortbildung, Leipzig/ Moskau 1985.

Zifonun, G., Zur Theorie der Wortbildung am Beispiel der deutschen Präfixverben, als: Linguistische Reihe 13, München 1973. 
